\title{
Isolated Persistent Fifth Aortic Arch with Right-sided Aortic Arch
}

\author{
Jiunn-Ren Wu, M.D., Chaw-Chi CHIU, M.D.,* \\ Young-Tso LiN, M.D.,* \\ and Teh-Yang Huang, M.D.
}

\section{SUMmary}

A persistent fifth aortic arch was recognized in a 4 -year-old boy by echocardiography, magnetic resonance imaging and angiocardiography. The case was unique in at least two respects: right-sided aortic arch, and systemicto-pulmonary connection without intracardiac lesion. This rare type of anomaly was correctly diagnosed and successfully repaired by patch closure of the arch. This is the first report of this combination of lesions in the Orient. (Jpn Heart Journal 36: 813-817, 1995)

Key words: Fifth aortic arch Right-sided aortic arch Systemic-topulmonary connection

$\mathrm{P}$ ERSISTENT fifth aortic arch (PFAA) is an extremely rare congenital cardiovascular malformation. It is an abnormal arterial branch originating from the distal ascending aorta proximal and opposite to the ostium of the innominate artery. ${ }^{1)}$ Most cases are associated with severe cardiac malformations and diagnosed fortuitously. In a previous review, only 3 of the 21 cases presented as a right-sided aortic arch. ${ }^{2)}$ Recently, we encountered a child with PFAA associated with a right-sided arch without another anomaly. We describe here, for the first time in the literature, its magnetic resonance imaging features. The anomaly was diagnosed and successfully repaired by patch closure of the arch.

\section{Case Report}

A 4-year-old boy was admitted to hospital because of heart murmur and increasing dyspnea. He had leg edema and mild cyanosis of the lips in the newborn period. Digoxin and diuretics were prescribed for the treatment of congestive heart failure for more than one year. On physical examination, he

\footnotetext{
From the Departments of Pediatrics and *Surgery, School of Medicine, Kaohsiung Medical College Kaohsiung 807, Taiwan, R.O.C.

Address for correspondence: Jiunn-Ren Wu, M.D., Department of Pediatrics, Kaohsiung Medical College, 100 Shih-Chuan 1st Road, San-Ming District, Kaohsiung 807, Taiwan.

Received for publication June 28, 1995

Accepted July 17, 1995.
} 


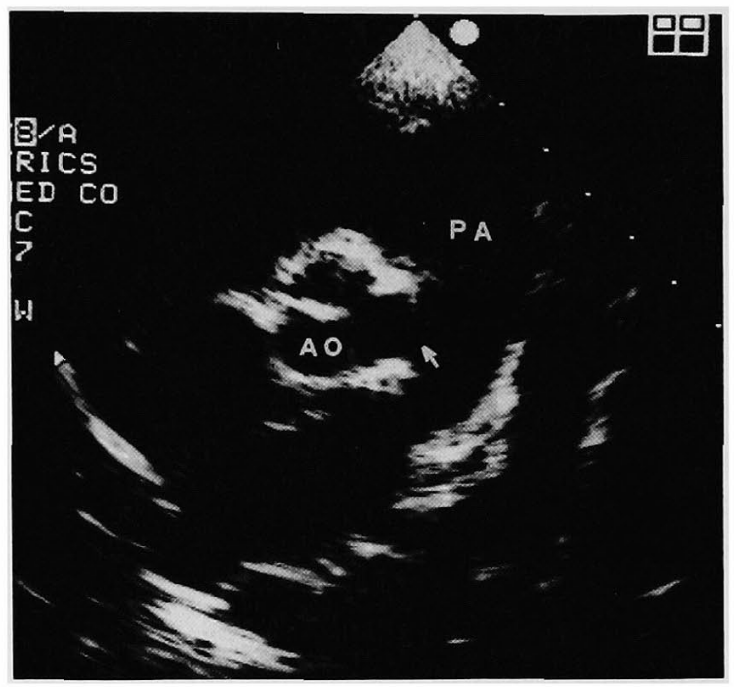

Figure 1. A 2-dimensional echocardiogram at the left upper sternal level demonstrated a large defect (arrow) along the aorta (AO) and the pulmonary artery (PA) border.

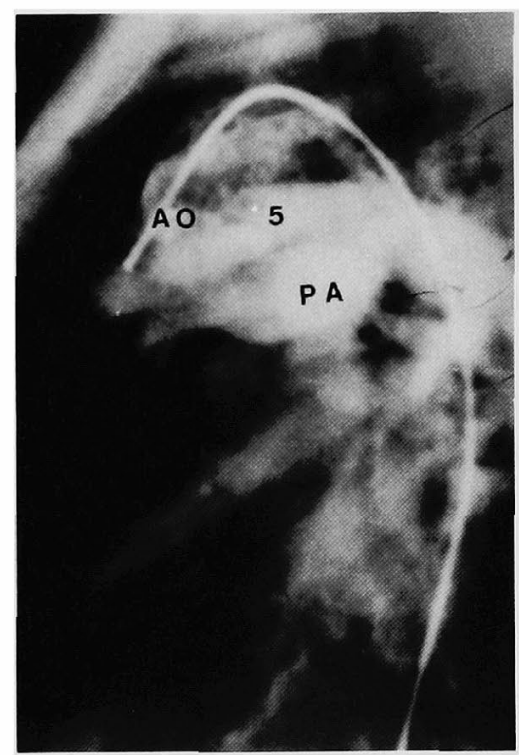

Figure 2. An aortogram of the left lateral projection revealed early systemic to pulmonary opacification via the 5 th aortic arch in the early films.

weighed $14.5 \mathrm{~kg}$. A grade 4/6 continuous machinery murmur was heard maximally at the base. An electrocardiogram showed a frontal $\mathrm{QRS}$ axis of $50^{\circ}$, left atrial hypertrophy and biventricular hypertrophy. Chest roentgenogram revealed moderate cardiomegaly, dilated pulmonary arterial segment, and increased pul- 

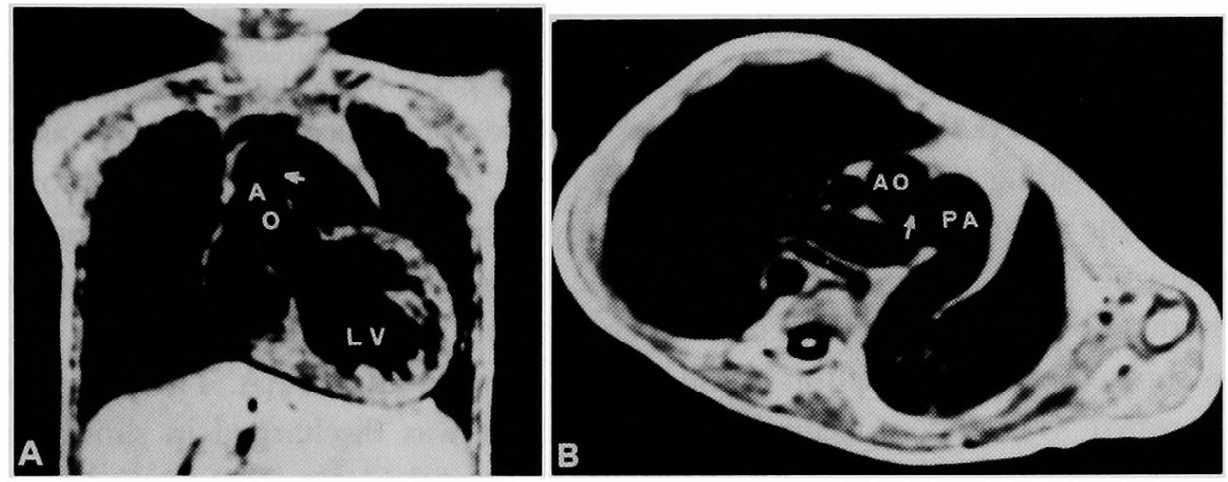

Figure 3. The magnetic resonance imaging findings. A: Coronary plane demonstrated a defect (arrow) at the left side of the distal ascending aorta (AO). B: An abnormal vessel connected between the ascending aorta and the pulmonary artery (PA) by an axial plane.

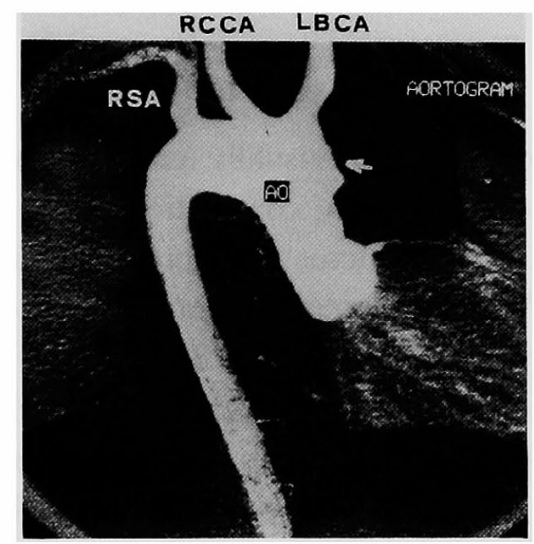

Figure 4. Postoperative aortogram showed a right-sided aortic arch, 3 branches from the aortic arch, and a "patch" shadow (arrow) at the distal ascending aorta and just proximal to the left brachiocephalic artery (LBCA). RCCA = right common carotid artery; RSA = right subclavian artery.

monary arterial vascularity. Color Doppler two-dimensional echocardiography at the left upper sternal level demonstrated a large "defect" with an aortic-to-pulmonary shunt along the aorta and the pulmonary artery border (Figure 1). Cardiac catheterization showed a right-sided aortic arch. The oxygen saturation rose $24 \%$ from the right ventricle to the pulmonary artery. The pulmonary to systemic flow ratio was $>4$. There was pulmonary hypertension with the pulmonary to systemic pressure ratio being as high as 0.9 . An aortogram of the left lateral projection revealed a systemic-to-pulmonary shunt at the level of the distal ascending aorta (Figure 2). The magnetic resonance imaging of the coronal plane demonstrated the abnormal "defect" at the left side of the distal ascending aorta (Figure 3-A). An abnormal vascular connection between the ascending aorta and 
pulmonary artery was also well visualized by an axial plane (Figure 3-B).

Corrective surgery with ligation of the PFAA was planned. At surgery, however, the PFAA could not be ligated because of technical difficulties in assessing and dividing the huge, short vessel of the PFAA. Under cardiopulmonary bypass, the pulmonary artery was vertically incised. The distal PFAA opening, measuring $2.5 \times 2.5 \mathrm{~cm}$, was found at the bifurcation of the pulmonary trunk. The diagnosis of PFAA could thus be confirmed ${ }^{3)} \mathrm{A} 3.0 \times 3.0 \mathrm{~cm}$ dacron patch was sutured near the junction of the ascending aorta and PFAA. The postoperative course was uncomplicated and the patient was discharged in satisfactory condition. Recatheterization was performed 6 months later. The hemodynamic studies were normal. An ascending aortogram in the frontal projection showed 3 branches from the right-sided aortic arch and a "patch" shadow at the distal ascending aorta and just proximal to the left brachiocephalic artery (Figure 4).

\section{Discussion}

All reported cases of PFAA are usually associated with severe cardiac malformations such as pulmonary atresia, coarctation of the aorta, ventricular septal defect, tricuspid atresia, patent ductus arteriosus and others. ${ }^{2)}$ All 5 previously reported cases with PFAA and systemic-to-pulmonary connection were associated with pulmonary atresia. ${ }^{3-6)}$ To the best of our knowledge, our case is different from all other reported cases in that, except for a right-sided aortic arch, our patient had no other cardiac malformation. The PFAA in this case was anatomically a subway between the ascending aorta and the pulmonary artery and had hemodynamically a large left to right shunt with severe pulmonary hypertension. This case cannot be regarded as an aorto-pulmonary window as Gerlis and his colleagues $^{3)}$ reviewed the literature and pointed out that the only vascular structure that can originate from the ascending aorta and proximal to the brachiocephalic arteries is the PFAA.

This rare type of PFAA can be correctly detected by 2-dimensional Doppler echocardiography, angiocardiography and magnetic resonance imaging and can be successfully repaired.

\section{REFERENCES}

1. Van Praagh R, Van Praagh S: Persistent fifth arterial arch in man; congenital double-lumen aortic arch. Am J Cardiol 24: 279, 1969

2. Gerlis LM, Dickinson DF, Wilson N, Gibbs JL: Persistent fifth aortic arch; a report of two new cases and a review of the literature. Int J Cardiol 16: 185, 1987

3. Gerlis LM, Ho SY, Anderson RH, Costa PC: Persistent 5th aortic arch; a great pretender; three new covert cases. Int J Cardiol 23: 239, 1989

4. Freedom RM, Silver M, Miyamura $\mathrm{H}$ : Tricuspid and pulmonary atresia with coarctation of the aorta; 
a rare combination possibly explained by persistence of the fifth aortic arch with a systemic-to-pulmonary arterial connection. Int J Cardiol 24: 241, 1989

5. Moes CAF, Freedom RM: Rare types of aortic arch anomalies. Pediatr Cardiol 14: 93, 1993

6. Yoo SJ, Moes CAF, Burrows PE, Molossi S, Freedom RM: Pulmonary blood supply by a branch from the distal ascending aorta in pulmonary atresia with ventricular septal defect; differential diagnosis of fifth aortic arch. Pediatr Cardiol 14: 230, 1993 